\title{
Anders Kjellberg Radikalisierung oder Japanisierung? Die Entwicklung des "schwedischen Modells« industrieller Beziehungen in den achtziger Jahren
}

Ende der sechziger Jahre hat in der schwedischen Gewerkschaftsbewegung eine Phase deutlicher Radikalisierung begonnen. Der Kontrast zu den vorangegangenen zwei bis drei Jahrzehnten war groß. Bis dahin orientierten die Gewerkschaften auf die Höhe der effektiven Produktion als Voraussetzungen für Lohnerhöhungen, ohne in einzelwirtschaftliche Prozesse zu intervenieren. Dies änderte sich Ende der sechziger Jahre mit Forderungen nach Mitbestimmung und Humanisierung des Arbeitslebens. Diese Forderungen jedoch waren primär an die staatliche Gesetzgebung gerichtet. In dem Versuch der Instrumentalisierung des Staates - und insoweit kann man von Radikalisierung sprechen - lag ein Bruch mit der bisherigen Praxis des »schwedischen Modells« industrieller Beziehungen, nämlich der Tradition, daß Gewerkschaften und Arbeitgeber ihre Beziehungen über Abkommen selbst regeln, um staatliche Maßnahmen so zu verhindern oder zu erübrigen. Die verstärkte Einbeziehung des Staates resultierte in Gesetze über Mitbestimmung, Humanisierung des Arbeitslebens und Arbeitnehmerfonds, welche die Positionen der Beschäftigten - in unterschiedlichem Ausmaße - verbesserten. Bereits Mitte der siebziger Jahre und bereits bevor etwa das Mitbestimmungsgesetz in Kraft trat, veränderten sich wesentliche Rahmenbedingungen. Etwa gleichzeitig mit der Regierungsübernahme durch die bürgerlichen Parteien im September 1976 verschlechterte sich die Wirtschaftslage drastisch und es kam zum Personalabbau in vielen Unternehmen.

Im folgenden soll nun - vor dem Hintergrund veränderter Kräfteverhältnisse in den Sozialbeziehungen - die Wirksamkeit und Wirkung der »staatlichen Strategie« der schwedischen Gewerkschaftsbewegung untersucht werden. Dafür sollen zunächst einige Angaben zum Kräfteverhältnis Arbeit und Kapital und zu wesentlichen Institutionen industrieller Beziehungen in Schweden dargestellt werden.

\section{Die Entwicklung des Kräfteverhältnisses Arbeit-Kapital}

Bürgertum und Arbeitgeber haben im Lauf der siebziger Jahre eine allgemeine Gegenoffensive eingeleitet, die durch die veränderten wirtschaftlichen und politischen Konjunkturen auf internationaler Ebene ermöglicht wurde. Dies bedeutet allerdings nicht, daß Schweden im gleichen Maße wie andere Länder von der Entwicklung auf internationaler Ebene betroffen war. In einigen Punkten sind die negativen Auswirkungen für Schweden geringer als für andere Länder ausgefallen. So ist die offene Arbeitslosigkeit im internationalen Vergleich weiterhin niedrig: $1,6 \%$ in den Jahren 1975 und 1976,2,2\% in 1978, 2,0\%1980, 2,5\% 1981 und $3,1 \%$ 1982. ${ }^{2}$ So sind auch keine negativen Auswirkungen auf die organisatorische Stärke der Arbeiterklasse - gemessen am gewerkschaftlichen Organisationsgrad - festzustellen. Ganz im Gegenteil ist der gewerkschaftliche Organisationsgrad auch nach Mitte der siebziger Jahre stark angestiegen: für Arbeiter von 77 \% im Jahre 1974 auf 85\% 1981 und für die Lohnabhän- 
gigen insgesamt von ebenfalls $77 \%$ im Jahre 1974 auf $86 \% 1981$ (vgl. Tabelle 1). Teilweise läßt sich dieser Anstieg im Organisationsgrad dadurch erklären, daß in Schweden wie Dänemark und in der Praxis auch Belgien (wo die Auszahlung von Arbeitslosengeld an die Gewerkschaften delegiert ist) die Arbeitslosenkassen in den Händen der Gewerkschaften liegen.

Kennzeichnend für die schwedische Situation ist, daß der gewerkschaftliche Organisationsgrad hier seit 1930 nie gesunken ist, und dies nicht einmal in für die Arbeiterbewegung ungünstigen politischen Konjunkturen, wie etwa dem Kalten Krieg. Der Kontrast zur Entwicklung vor allem in Italien, Frankreich und den USA ist auffällig. In diesen Ländern sank der Organisationsgrad seit Ende der vierziger bzw. Anfang der fünfziger Jahre stark. Gemeinsam mit Dänemark, Norwegen, Österreich und Belgien bildet Schweden während der ganzen Nachkriegszeit eine Ländergruppe mit hohem gewerkschaftlichem Organisationsgrad (vgl. Tabelle $1 \mathrm{im}$ Anhang). Australien und Großbritannien stellen hier eineZ Zischengruppe dar, während die Bundesrepublik, die Niederlande, Italien, Frankreich und die USA zur unteren Gruppe gehören.

Zum hohen gewerkschaftlichen Organisationsgrad in den skandinavischen Ländern hat beigetragen, daß die Gewerkschaftsbewegung hier eine solche Stärke erreicht hat, daß sie den Arbeitgebern wesentliche Konzessionen abringen konnte. Ausgangspunkt für diese Entwicklung in Schweden war das Zentralabkommen von 1938 (Vertrag von Saltsjöbaden) zwischen dem Gewerkschaftsbund LO und dem Arbeitgeberverband SAF, das u.a. die Anerkennung des gewerkschaftlichen Organisationsrechts vorsah. Da auch die Arbeitgeberseite zentral organisiert war, bekam dieses Abkommen weitreichende Bedeutung. Schon zu Beginn dieses Jahrhunderts gab es einige Vorläufer dieser Vereinbarung, nämlich die Metallvereinbarung des Jahres 1905 und der sog. 'Dezember-Kompromiß' zwischen LO und SAF des Jahres 1906, bei dem beide Parteien das Recht der Organisierung der Interessen anerkannten. Im übrigen ist der hohe und zunehmende Grad der Organisierung durch die gut ausgebaute gewerkschaftliche Vertretung auf Arbeitsplatzebene stabilisiert und gefördert worden. Die Stärke der Arbeiterbewegung dokumentiert sich auch in dem Umstand, daß Arbeiterparteiregierungen selbst in der Periode des Kalten Krieges Bestand haben konnten.

Von wesentlicher Bedeutung für das Kräfteverhältnis zwischen Kapital und Arbeit ist, daß die Arbeitgeberseite in den skandinavischen Ländern äußerst gut organisiert ist. Ihre wohldisziplinierten Arbeitgeberverbände verfügen über gewaltige Streikfonds und besitzen ein weitreichendes Aussperrungsrecht. ${ }^{2}$ Auch die Wirtschaftsstruktur Schwedens verleiht den Arbeitgebern wichtige Machtpositionen. Durch weitreichende Eigentumskonzentration kombiniert mit einer stark multinationalen Ausrichtung der Großunternehmen werden Regierungen gleich welcher politischen Ausrichtung gezwungen, auf die vorherrschenden Wirtschaftsinteressen Rücksicht zu nehmen. Zu erwähnen ist, daß bei Großunternehmen wie Volvo, Elektrolux, Ericsson, SKF, Sandvik und Atlas Copco der gesamte Absatz im Ausland(Export plus Auslandsproduktion) zwischen Dreiviertel und Neunzehntel des Gesamtumsatzes ausmacht. ${ }^{3}$

Ein anderer Umstand, der bei der Beurteilung des Stärkeverhältnisses in den Kapital-Arbeitsbeziehungen beachtet werden muß, ist der Grad der Mitgliederaktivitär und das Mobilisierungspotential der Gewerkschaften. Hier muß festgestellt werden, daß der Typus kooperativer Gewerkschaften, wie er in Schweden in der Nachkriegszeit dominierte, aufgrund der niedrigen Konflikthäufigkeit geringere Anforderungen an die Solidarität der Mitglieder stellt als ein Gewerkschaftstypus, der stärker auf Konfrontation mit den Arbeitgebern angelegt ist. Bis zu den dreißiger Jahren dominierte im übrigen in Schweden der Konfrontationstyp. In 
den ersten Jahrzehnten des 20. Jahrhunderts befand sich Schweden unter den Ländern mit hoher Konflikthäufigkeit und lang anhaltenden Arbeitskonflikten. ${ }^{4}$

Im Schweden der Nachkriegszeit ist es deshalb kaum überraschend, daß Probleme gewerkschaftlicher Inaktivität der Mitglieder auftreten. In den letzten Jahren ist dieses Problem in den Gewerkschaften zunehmend diskutiert worden (u.a. in den Berichten für den LO-Kongreß 1981). ${ }^{5}$ Das Problem hat sich verschärft, weil die neuen Gesetze der siebziger Jahre für die Gewerkschaften größere Aufgaben mit sich brachten und bessere Kenntnisse erforderlich waren. Von LO's Mitgliedern betrachteten sich $197817 \%$ als gewerkschaftlich aktiv, wobei $11 \%$ gewerkschaftliche Funktionen wahrnahmen. ${ }^{6}$ Die Rekrutierungsbasis der Funktionäre ist also nicht sonderlich groß.

Von Bedeutung für das Kräfteverhältnis Arbeit-Kapital sind weiterhin die Beziehungen zwischen gewerkschaftlicher und politischer Aktivität. Im internationalen Vergleich hat in Schweden traditionell ein sehr gutes Verhältnis zwischen der Gewerkschaftsbewegung und der sozialdemokratischen Partei bestanden. Auf der parlamentarischen Ebene unterstiitzt die kleine kommunistische Partei (ca. 5 \% der Stimmen) die Sozialdemokratie. Zusammen bilden sie den sog. sozialistischen Block. Diesem gegenüber steht der bürgerliche Block - so die allgemeine Bezeichnung - bestehend aus drei bürgerlichen Parteien. Im Verhältnis zur sozialdemokratischen Partei, die 44 Jahre lang hintereinander die Regierungsgewalt besaß (1932 bis 1976) fungierte die Gewerkschaftsbewegung als Antriebsfaktor. Von zentraler Bedeutung war in dieser Hinsicht die Herausbildung der "solidarischen Lohnpolitik" in der Nachkriegszeit.

Die schwedische Gewerkschaftsbewegung ist traditionell stärker als die Gewerkschaften anderer Länder darum bemüht, große Lohnunterschiede zwischen den Berufs- und Tätigkeitsgruppen bzw. den Branchen und Sektoren der Volkswirtschaft zu vermeiden und einen Interessenausgleich innerhalb der Lohnarbeiterschaft zu organisieren. Hierin unterscheidet sie sich markant von beispielsweise der deutschen oder der französischen Gewerkschaftsbewegung, die immer noch in erster Linie die Facharbeiter organisieren. ${ }^{7}$ Bereits um 1930 war die Angleichung der Löhne bei den Arbeitern der schwedischen Maschinenbau- und Metallverarbeitungsindustrie verglichen mit anderen Ländern weit vorangeschritten. ${ }^{8}$ Die solidarische Lohnpolitik der Nachkriegszeit hatte jedoch eine Kehrseite. Weil die Löhne unter dem Gesichtspunkt der Lohnangleichung in den wenig profitablen Niedriglohnbranchen wie der Textil- und Konfektionsindustrie, relativ am stärksten erhöht werden mußten, legten viele Betriebe hier die Produktion still, und die Arbeiter mußten eine Anstellung in den expandierenden Industriezweigen suchen, die ihrerseits davon profitierten, daß die Löhne dort relativ geringe Zuwachsraten hatten. Um die Mobilität der Arbeitskraft in geographischer und beruflicher Hinsicht zu erhöhen, und damit diesen Effekt der solidarischen Lohnpolitik zu verstärken, bildete sich die sog. aktive Arbeitsmarktpolitik als ein weiteres zentrales Element des »schwedischen Modells« heraus. Deren Verwaltungsorgan wurde die AMS, deren Perscnal - in Widerspruch zu den traditionellen Prinzipien der schwedischen Staatsverwaltung zu einem großen Teil aus der Gewerkschaftsbewegung rekrutiert wurde. ${ }^{9}$

Die solidarische Lohnpolitik verfolgte mehrere Ziele. In Kombination mit der aktiven Arbeitsmarktpolitik sollte sie einerseits wirtschaftliches Wachstum stimulieren, u.a. durch $\mathrm{Be}$ friedigung des Arbeitskräftebedarfes der Industrie. Andererseits fungierte sie als eine Art Einkommenspolitik in Eigenregie der Gewerkschaften. Dadurch hat die Gewerkschaftsbewegung es vermeiden können, in die "Fallen« einer Einkommenspolitik zu geraten, in die die englischen und holländischen Gewerkschaften zeitweilig geraten sind - mit negativen 
Auswirkungen auf den Zusammenhalt der Arbeiterbewegung (Großbritannien) bzw. auf die Entwicklung der gewerkschaftlichen Mitgliederzahlen (Niederlande in den fünfziger und sechziger Jahren). ${ }^{10}$ Damit ist auch die Bedeutung der tarifautonomen Regelungen als charakteristisches Moment des »schwedischen Modells« verstärkt worden. So kommt es auch nicht von ungefähr, daß die Konzeption der solidarischen Lohnpolitik und der aktiven Arbeitsmarktpolitik von den Wirtschaftsexperten des zentralen Gewerkschaftsbundes, Gösta Rehn und Rudolf Meidner, entwickelt wurden. Indem die Gewerkschaften staatliche Einkommenspolitik unnötig machten, vermieden sie es, in den Augen ihrer Mitglieder alsüberflüssig zu erscheinen, während gleichzeitig ihr Eintreten für wirtschaftliches Wachstum reale Lohnerhöhungen erleichterten. ${ }^{11}$

Ende der sechziger Jahre kam es zu einer Kursänderung der Gewerkschaften. Innerhalb der LO war es vor allem die Metallarbeitergewerkschaft, die mit entsprechenden Initiativen hervortrat. Als die bis 1978 (als die Kommunalarbeitergewerkschaft sie größenmäßig überholte) größte Organisation innerhalb von LO und als die für die für Schweden wirtschaftlich äußerst wichtigen Metall- und Maschinenbauindustrie organisatorisch zuständige Gewerkschaft war sie in besonderem Maße von den intensivierten Strukturrationalisierungen und Betriebsstillegungen der sechziger Jahr betroffen, wobei deutlich Lücken in ihren Handlungs- und Informationsrechten sichtbar geworden waren. ${ }^{12}$ Auch kamen jetzt Probleme belastender Arbeitsbedingungen klarer zum Vorschein. Die zuvor ungeteilt positive Einstellung zu wirtschaftlichem Wachstum und technischer Entwicklung, die einseitig unter dem Verteilungsgesichtspunkt als Voraussetzung für Lohnerhöhung und soziale Reformen gesehen und deren negative Folgen auf der Ebene der Betriebe übersehen wurde, wurde nun überprüft. Wirtschaftswachstum und technische Entwicklung wurden danach zwar auch weiterhin als notwendig betrachtet. Neu war aber, daß man sich nun ernsthaft mit den negativen Folgen auseinandersetzte. Der große Streik in den Eisenerzbergwerken von LKAB in Kiruna 1969 diente als Signal. ${ }^{13}$ Das radikalisierte ideologisch-politische Klima am Ende der sechziger Jahre barg zudem das Risiko für die Gewerkschaften, nicht nur im Verhältnis zu ihren Mitgliedern, sondern auch in der öffentlichen Debatte in eine defensive Position zu geraten. Die Notwendigkeit zu einer Positionsänderung gewerkschaftlicher Politik zeigte sich schließlich an der Welle wilder Streiks in der Maschinenbauindustrie, die 1970 auf den LKAB-Streik folgten.

Vor dem Hintergrund dieser Entwicklung änderte die Führung von LO ihren Kurs zugunsten einer Politik der Mitbestimmung, der Humanisierung des Arbeitslebens und der Beschäftigungssicherheit.

\section{Der Übergang zur Strategie der gewerkschaftsunterstützenden Gesetzgebung}

Eine Fortführung der Politik von Kooperationsabkommen zwischen den Zentralverbänden der Gewerkschaften und Arbeitgeber schien im Hinblick auf diese Reorientierung wenig erfolgversprechend. Stattdessen wurden in den siebziger Jahren eine Reihe von Gesetzen zur Regelung der Kapital-Arbeitsbeziehungen verabschiedet, u.a. das Kündigungsschutzgesetz (1974) und das Mitbestimmungsgesetz (1976). Das letzte in der Reihe dieser Gesetze stellt der Beschluß des Reichstages im Dezember 1983 dar, Arbeitnehmerfonds einzurichten. Dies emsige Verabschieden von Gesetzen beinhaltet einen Bruch mit dem traditionellen Charakterzug des schwedischen Modells, der darin bestand, daß die»Partner« des Arbeitsmarktes ihre 
Beziehungen selbst durch Abkommen regeln, um Staatseingriffe zu verhindern in der Überzeugung, daß die Zusammenarbeit zu den besseren Ergebnissen führe.

Die veränderte Rolle des Staates sollte jedoch nicht überschätzt werden. ${ }^{14} \mathrm{Zum}$ ersten haben sozialdemokratische Regierungen auch vor den siebziger Jahren zu der Gesetzgebung gegriffen, wenn es um Fragen ging, die im Prinzip auch durch Abkommen zwischen den Tarifparteien hätten geregelt werden können, wie z.B. die Regelung von Urlaubszeiten usw. Zum anderen kommt den gewerkschaftlichen Organisationen auch nach den neuen Gesetzen eine bedeutsame Rolle zu, was ganz der schwedischen Tradition entspricht. Die Kontinuität der bisherigen, das »schwedische Modell « tragenden Einstellungen läßt sich insbesondere an der Konzeption des Mitbestimmungsgesetzes zeigen. Das Gesetz enthält an sich keine Vorschriften, die ein Recht auf Mitbestimmung garantieren; es enthält nur Regeln in bezug auf ein verstärktes Verbandlimgsrecht der Gewerkschaft. Daß das Gesetz auf diese Weise konstruiert wurde, geschah explizit auf gewerkschaftlichen Wunsch hin; die Gewerkschaftsbewegung wollte die Rolle der Verhandlungen aufrecht erhalten. So war im Gesetz vorgesehen, daß die gesetzlichen Regelungen durch Abkommen zur Mitbestimmung zwischen den Tarifparteien ergänzt und konkretisiert werden. Insoweit geht das Mitbestimmungsgesetz einen Schritt weiter als die Abkommen über Betriebsaussschüsse von 1946 und 1966, das es ersetzte, indem nun das Moment der direkten Verhandlungen zwischen Gewerkschaft und Arbeitgebern betont wurde. Es ist denn auch wiederholt darauf hingewiesen worden, daß das Mitbestimmungsgesetz insofern inkonsequent sei, als es zustande gekommen sei, weil die Gewerkschaftsbewegung die bisherige Politik der Verhandlungen und Abkommen als unzureichend betrachtete, das Gesetz nun aber im Großen und Ganzen selbst nichts anderes sei als eine Anweisung der Tarifparteien für Verhandlungen und Abkommen. In dem Gesetz drückt sich mithin ein Idealismus aus, Machtverschiebungen im Verhältnis von Kapital und Arbeit im Einverständnis über Verträge ohne irgendwelche Druckmittel erreichen zu können.

\subsection{Erfabrungen mit der Umsetzung des Mitbestimmungsgesetzes}

Unter den veränderten wirtschaftlichen und politischen Bedingungen seit Ende der siebziger Jahre war es für die Arbeitnehmerseite schwer, Vorteile aus dem Mitbestimmungsgesetz zu ziehen. Erst im Frühjahr 1982 wurde diese Umsetzungsvereinbarung zwischen SAF auf der einen, LO und PTK (Verhandlungskartell der Angestellten in der Privatindustrie) auf der anderen Seite abgeschlossen. Die Umsetzungsvereinbarung der maschinenbau- und metallverarbeitenden Industrie, die am 1. Juli 1983 in Kraft trat, basiert im wesentlichen auf dieser zentralen Vereinbarung. ${ }^{15}$

Vor Inkrafttreten des Mitbestimmungsgesetzes am 1.1.1977 waren die Erwartungen auf gewerkschaftlicher Seite hoch, daß dem Arbeitgeber jetzt das alleinige Recht auf »hire and fire« der Arbeitskraft sowie auf den innerbetrieblichen Arbeitseinsatz genommen würde. Die wirtschaftliche Krise und die verstärkte Verhandlungsposition der Arbeitgeber während der bürgerlichen Regierungsperiode und deren Weigerung, während dieser Zeit Umsetzungsabkommen abzuschließen, machte derartige Hoffnungen zunichte. Die per Gesetz verordnete Verhandlungspflicht wird jetzt - auch in Gewerkschaftskreisen - als die "Hupe wezeichnet, welche die Arbeitgeber benutzen, bevor sie ihre Beschäftigten z.B. durch Betriebsstillegungen oder Personalabbau »überfahren«. Nicht selten hat sich der Einfluß der Gewerkschaften darauf beschränkt, auszuwählen, wer zuerst entlassen werden soll. Paradoxerweise 
hat dies das 1974 verabschiedete Gesetz über Kündigungsschutz praktisch außer Kraft gesetzt. Im Gegensatz zu den Bestimmungen dieses Gesetzes über eine nach Seniorität ansteigende Beschäftigungssicherheit hat die durch die Mitbestimmungsgesetzgebung konstituierte Verhandlungssituation dazu geführt, daß jüngere Arbeiter ihre Arbeitsplätze behalten konnten, während diejenigen mit den längsten Anstellungszeiten zuerst gehen mußten. Nur in den Fällen, in denen die Unternehmensleitung unter Druck war und schnelle Entscheidungen benötigte, hatte die Gewerkschaft gelegentlich die Möglichkeit, auf Entscheidungen über Betriebsstillegungen, Entlassungen, Produktionsverlagerungen u.ä. wesentlich Einfluß zu nehmen. ${ }^{16}$ In einer Reihe von staatlichen Unternehmen (z.B. Berol-Chemie) war der gewerkschaftliche Einfluß in dieser Hinsicht allerdings beträchtlich größer. ${ }^{17}$

Die zentrale Umsetzungsvereinbarung zwischen SAF und LO/PTK von 1982 berührt drei Gebiete: die Entwicklung der Arbeitsorganisation, die technische Entwicklung und Fragen der wirtschaftlichen Unternehmensführung, nicht jedoch Personalfragen. Das Abkommen regelt auch Fragen der Forschung, des Einsatzes von externen Arbeitnehmerberatern und der gewerkschaftlichen Arbeit in Konzernen. Bis jetzt ist nur in einem einzigen Unternehmen der Maschinenbau- und metallverarbeitenden Industrie eine lokale Umsetzungsvereinbarung verabschiedet worden, nämlich bei Volvo, Schwedens größtem Unternehmen. ${ }^{19}$ Die Neuigkeit im Verhältnis zum Zentralabkommen besteht hier darin, daß die Gewerkschaft bei der Einführung neuer Techniken in einem frühen Stadium eingeschaltet wird, sobald es um Fragen der Gestaltung der Arbeitsplätze geht. Außerdem hat sich das Unternehmen dazu verpflichtet, überzähliges Personal, das nicht versetzt werden kann, umzuschulen. Die Verhandlungsführer der Gewerkschaft weisen jedoch darauf hin, daß auch dieses Abkommen nichts an den bestehenden Machtverhältnissen geändert hat. Im Falle der Nichteinigung hat nach wie vor der Arbeitgeber die Entscheidungsgewalt. Zentrale Probleme gegenwärtiger Arbeitspolitik, wie sie etwa mit der Einführung neuer Techniken verbunden sind, lassen sich - so auch die überwiegende Einschätzung der Teilnehmer einer Konferenz der Metallarbeitergewerkschaft in Stockholm über neue Techniken - nicht lösen. ${ }^{20}$

\subsection{Erfabrungen mit Maßnabmen zur Humanisierung des Arbeitslebens}

In der Frage der Arbeitsbedingungen ließ sich die seit Ende der 60er Jahre veränderte Einstellung der Gewerkschaftsbewegung am deutlichsten beobachten. Ausgangspunkt war hier u.a. eine Untersuchung über Arbeitsbedingungen, die LO 1968/69 durchführte. Diese zeigte, daß $82 \%$ der Gewerkschaftsmitglieder unter arbeitsbedingten Belastungsfaktoren litten und immerhin $42 \%$ derartigen Belastungen in besonders hohem Grade ausgesetzt waren. ${ }^{21}$ Einige Jahre später erschien ein Bericht, demzufolge etwa $30 \%$ der Befragten an ihrem Arbeitsplatz von Streßbelastungen betroffen waren. Nach einer Untersuchung im Jahre 1980 litten $87 \%$ unter arbeitsbedingten Belastungen, davon $49 \%$ in hohem Grade. Dies bedeutet eine Steigerung um 5 bis $7 \%$ seit der o.a. Untersuchung von 1968/69. Ein Teil der Erklärung für diesen Zuwachs mag darin liegen, daß u.a. die technische Entwicklung tatsächliche Verschlechterungen der Arbeitsumwelt mit sich gebracht hat. Vieles weist darauf hin, daß dies insbesondere in bezug auf chemische und psycho-soziale Faktoren der Fall ist. Auf dem Gebiet der Chemie ist eine Reihe neuer Stoffe und Produkte eingeführt worden, die zudem auf immer mehr Gebieten des Arbeitslebens Verwendung finden.

Ein anderer Teil der Erklärung wird an den vermutlich besseren Kenntnissen, veränderten 
Bewertungen und den zunehmenden Forderungen an die Arbeitsumwelt liegen, die u.a. auch in den neuen Gesetzen und Vereinbarungen ihren Niederschlag gefunden haben.

Die höhere Priorität, die den Problemen der Arbeitsumwelt seit Ende der 60er Jahre beigemessen wurde, kam 1974 in einem veränderten Arbeitsschutzgesetz zum Ausdruck; dieses wurde 1978 durch ein umfassenderes Arbeitsumweltgesetz ersetzt. Zusätzliche Abkommen zwischen den Tarifparteien zum gleichen Problembereich wurden 1967 und 1981 getroffen. Ein für die Gewerkschaften wichtiger Erfolg bestand darin, daß die gewerkschaftlichen Schutzbeauftragten durch das Gesetz 1974 das Recht erhielten, vorläufig die Arbeit einstellen zu lassen, wenn eine »unmittelbare oder ernste Gefahr für das Leben oder die Gesundheit des Arbeitnehmers« vorliegt. In vielen Fällen hat bereits die Androhung der Arbeitseinstellung ausgereicht, um den Arbeitgeber zu Maßnahmen zu veranlassen. Es wurden regionale Arbeitsschutzbeauftragte eingestellt, um die Arbeitsplätze in Kleinbetrieben zu überwachen. Außerdem erhielten die Schutzbeauftragten das Recht und die Pflicht, an der Planungsarbeit teilzunehmen. Durch das Arbeitsumweltabkommen von 1967 bekamen die Arbeitnehmer (Arbeiter und Angestellte) die Mehrheit in den betrieblichen Arbeitsschutzkommitees, deren Befugnisse zudem erweitert wurden.

Bei der Entstehung des Arbeitsumweltgesetzes, das am 1. Juli 1978 in Kraft trat, folgte die bürgerliche Regierung im Großen und Ganzen den Vorschlägen, die von einer damals noch von der sozialdemokratischen Regierung abgestellten staatlichen Untersuchungskommission zu Problemen der Arbeitsumwelt vorgelegt worden waren. Das Gesetz ist ein Rahmengesetz, das der staatlichen Aufsichtsinstanz für Arbeitsschutzfragen das Recht gibt, selbstständig juristisch bindende Vorschriften zu erlassen, auch mit Straffolge. Zuvor waren die Anweisungen, die das Arbeitsschutzamnt erließ, nicht bindend, hatten also eher den Charakter von Ratschlägen; aber auch nun wurden bindende Vorschriften nur in solchen Fällen erlassen, in denen die Einhaltung leicht zu kontrollieren und zu erfüllen war. ${ }^{22}$ Das neue Gesetz beinhaltete, daß das Arbeitsschutzamt und die Inspektionsbehörde vor Ort sowohl ratgebend wie kontrollierend tätig werden können. Man sah hierin keinen Gegensatz. In der Praxis haben sich Kooperation mit dem Arbeitgeber und Beratung allerdings gegenüber der Verwendung rechtlicher Sanktionsmittel durchgesetzt. Ohnehin kann der Arbeitgeber erst nachdem die örtliche Inspektion unter Strafandrohung in Form von Verboten oder Auflagen interveniert hat bestraft werden.

Eine kürzlich von Lundberg vorgenommene Untersuchung über die Tätigkeit der Inspektionsbehörden zeigt, daß je nach Region sehr unterschiedliche Verhaltensweisen dominieren. Ein Teil von ihnen ist passiv und vermeidet so lange wie möglich $Z$ wangsmaßnahmen, ein anderer Teil vertritt eine härtere Linie und hat eher Polizeicharakter ${ }^{23}$ In jenen Fällen, in denen sich die betrieblichen Schutzbeauftragten beim zentralen Arbeitsschutzamt darüber beschwerten, daß die örtlichen Inspektionsbehörden zu milde verfahren seien, konnten sie dort nur schwer Gehör finden. ${ }^{24}$ Dagegen hatten die Arbeitgeber wesentlich größere Erfolge, sobald sie sich gegen Behördenbeschlüsse zur Wehr setzten. Im Ganzen gesehen dominiert stark das Prinzip der Freiwilligkeit, oder anders ausgedrückt: die legalistische Linie konnte sich u.a. wegen der Politik des Arbeitsschutzamtes, das Mittel der Verbote und Auflagen nur sparsam zu verwenden, kaum durchsetzen. ${ }^{25}$ Auf lokaler Gewerkschaftsebene fordert die LO mehr Vorschriften mit mehr Sanktionscharakter, um die Arbeitgeber stärker zu präventiven Maßnahmen zu veranlassen. ${ }^{27}$ Kürzlich hat der Justizombudsmann der schwedischen Regierung, dessen Aufgabe darin besteht, die Staatsverwaltung zu überwachen, nach einer routinemäßigen Inspektion eines Distrikts einen Bericht veröffentlicht, in dem kritisiert wird, daß 
Verstöße gegen mit Strafe zu ahndenden Vorschriften nicht zur Anklageerhebung bei der Staatsanwaltschaft geführt hätten. ${ }^{28}$ Dieses Beispiel illustriert, daß nicht einmal die existierenden Vorschriften effektiv sind, sondern die Tradition der Zusammenarbeit noch immer durchschlägt.

Jede Analyse der Möglichkeiten der Beschäftigten, ihre arbeitsumweltbezogenen Forderungen durchzusetzen, bliebe unvollständig, wenn man nicht die betriebliche gewerkschaftliche Arbeitsschutzorganisation betrachtet. ${ }^{29}$ Im Unterschied zu den meisten kontinentaleuropäischen Ländern (besonders bis zum Ende der 60er Jahre) besaßen die schwedischen Gewerkschaften seit langem eine ausgebaute Organisation auf Arbeitsplatzebene, die aus sog. Gewerkschaftsclubs und gewerkschaftlichen Vertrauensleuten besteht. ${ }^{30}$

Im Gegensatz etwa zu den west deutschen Betriebsräten sind dies rein gewerkschaftliche Organe. Die sog. Arbeitsschutzkommitees setzen sich demgegenüber aus Vertretern sowohl der Arbeitgeber- wie der Arbeitnehmerseite zusammen, stets mit Arbeitnehmermehrheit. ${ }^{31} \mathrm{Die}$ Schutzbeauftragten fungieren als eine Art gewerkschaftliche Vertrauensleute in Fragen der Arbeitsumwelt. Vor allem in größeren Betrieben besteht eine enge Zusammenarbeit zwischen der Leitung der Gewerkschaftsclubs und den gewerkschaftlichen Vertretern in den Arbeitsschutzkommitees. Nach der LO-Untersuchung von 1980 liegen in Betrieben mit aktiven Schutzkommitees auch die besten Arbeitsbedingungen vor. Hier führt das Handeln der Gewerkschaften auch zu den deutlichsten Ergebnissen. ${ }^{32} \mathrm{Nur} 27 \%$ der Schuzbeauftragten geben an, daß ihre Gewerkschaft sich an die örtliche Inspektionsbehöde gewandt habe. ${ }^{33}$ Im LOBericht wird als Grund dafür angegeben, $» \mathrm{daß}$ man von der Inspektionsbehörde nicht erwartet, daß diese irgendeinen Beitrag bei der Durchsetzung von Maßnahmen leisten könne«. ${ }^{34}$ Die Erfahrungen im Bereich von Maßnahmen zur Humanisierung der Arbeit lassen also die Schlußfolgerung zu, daß lokale gewerkschaftliche Aktivitäten sich trotz der oben beschriebenen Gesetze als effizienter erwiesen haben als staatliche Inspektionen. Zum anderen hat die staatliche Gesetzgebung dazu geführt, daß die Stellung der gewerkschaftlichen Schutzbeauftragten in den 70 er Jahren erheblich gestärkt wurde.

\subsection{Die Gewerkschaftsforderung nach Arbeitnebmerfonds}

Die Gesetzgebung der 70er Jahre zur Arbeitsumwelt ist im Großen und Ganzen im Zeichen politischer Übereinstimmung im schwedischen Reichstag verabschiedet worden. Das gilt aber nicht für die Frage der Arbeitnehmerfonds. Dies hat zu einer weitgehenden Polarisierung sowohl auf politischer Ebene wie auf der Ebene der Arbeitsmarktparteien geführt. Zudem sind zeitweilig auch Gegensätze zwischen der sozialdemokratischen Partei und dem Gewerkschaftsbund deutlich geworden. Hierbei ist zu beachten, daß der Vorschlag der Arbeitnehmerfonds ursprünglich von LO kam und vorangetrieben wurde, während die Partei mäßigend gewirkt hat. Sie tat dies aus politischer Rücksicht, u.a. aus der Furcht heraus, Randgruppenwähler zu verlieren und um sich Zusammenarbeitsmöglichkeit mit den bürgerlichen Parteien und der Privatwirtschaft zu erhalten.

Durch die vom Reichstag angenommene Vorlage von Dezember 1983 verwirklicht Schweden nun als erstes Land in Europa Arbeitnehmerfonds. Dies, obgleich die Debatte über wirtschaftliche Demokratie durch die Abführung von Unternehmensgewinnen in Arbeitnehmerfonds in Schweden im Vergleich zu anderen Ländern erst relativ spät in Gang kam. ${ }^{35}$ Die erste Initiative ging vom LO-Kongreß 1971 aus, der eine Untersuchungskommission unter 
Leitung des LO-Wirtschaftsexperten Meidner einsetzte. Den Hintergrund bildete das Problem der »Übergewinne«, die als eine Folge der solidarischen Lohnpolitik in einigen Wirtschaftsbereichen entstanden waren. Um die Lohnforderungen in expansiven Wirtschaftsbereichen, vor allem der metallverarbeitenden und Maschinenbauindustrie zurückzuhalten, waren kompensierende gewerkschaftliche Maßnnahmen notwendig. Es war also kein Zufall, daß die Metallarbeitergewerkschaft hierzu die Initiative ergriff. Ein weiteres Motiv für die Forderung nach Arbeitnehmerfonds war, der in Schweden sehr weitgehenden privaten Machtkonzentration in der Wirtschaft entgegenzuwirken und die Vorstöße auf dem Gebiet der Mitbestimmung zu flankieren.

1975 legte die Meidner-Kommission einen Vorschlag vor, der in etwas veränderter Form im folgenden Jahr vom LO-Kongreß angenommen wurde. Gemäß diesem Vorschlag sollte jährlich ein Teil der Gewinne der Großunternehmen in kollektive Arbeitnehmerfonds überführt werden. Während des Wahlkampfes 1976, den die Sozialdemokraten nach 44-jähriger Regierungszeit verloren, spielte die Frage der Arbeitnehmerfonds eine herausragende Rolle. In der Folgezeit sind LO und Sozialdemokratie schrittweise in dieser Frage immer weiter zurückgewichen, während gleichzeitig die bürgerlichen Parteien und der Arbeitgeberverband SAF ihre Kampagne gegen die Fonds zunehmend intensivierten. Dadurch wurden Verständigungslösungen, wie sie die Sozialdemokraten anstreben, zunichte gemacht. Paradoxerweise erreichte die Anti-Fondskampagne von SAF vor der Wahl im September 1982, welche die Sozialdemokraten infolge der mißglückten Wirtschaftspolitik der bürgerlichen Regierung gewannen, und im Laufe des Jahres 1983 einen Hö hepunkt, obwohl die vorgelegten Vorschläge in der Frage der Kontrolle über die Produktion weiter abgeschwächt worden waren. "Auf zum Kampf gegen den Fondssozialismus« lautete dennoch die Schlagzeile auf der ersten Seite der "SAF-Tidningen«, der Zeitschrift des Arbeitgeberverbandes (Nr. 22/1983). Darunter wurde die Erklärung des Arbeitgeberpräsidenten referiert, daß den Fonds »mit allen gesetzlichen Mitteln entgegengearbeitet würde«, was einen eintägigen Unternehmerstreik und die Weigerung, die Arbeitgeberabgabe an den Staat zu bezahlen, einschließe. Die Unternehmer veranstalteten im Oktober 1983 in Stockholm eine der größten Demonstrationen der schwedischen Geschichte mit ca. 75000 Teilnehmern. Dies steht in starkem Kontrast zu der kümmerlichen Mobilisierung von gewerkschaftlicher und sozialdemokratischer Seite. Anstatt Unterstützung für ihre Forderungen zu mobilisieren, ist man hier seit Mitte der 70er Jahre unaufhörlich zurückgewichen.

Der Vorschlag, der schließlich als Gesetz angenommen wurde, beinhaltet, daß die Arbeitnehmer auf einen Teil ihrer Lohnsteigerungen verzichten sollen, die dannn durch zentral gelenkte Fonds für den Aktieneinkauf verwendet werden. ${ }^{34 a}$ Die führende bürgerliche Tageszeitung Dagens Nyheter kommentierte den endgültigen Fondsvorschlag auf ihrer Börsenseite wie folgt: »Ist man Kapitalist und Anhänger des Grundsatzes, daß die Macht über die Aktiengesellschaften in Privathänden verbleiben müssen, braucht man sich nicht sonderlich zu beunruhigen. Der Vorschlag ist harmlos und stumpf. Er zeigt keine Krallen. Er enthält keine Machtansprüche mehr. Weder vor noch nach 1990 werden die jetzt vorgeschlagenen Arbeitnehmerfonds denen die Macht nehmen, die sie heute besitzen.« (Dagens Nyheter vom 16.10.1983)

Nichtsdestoweniger bedeuten die Fonds sogar in der nun verwirklichten Form etwas Neues im Vergleich zu der traditionellen gewerkschaftlichen und sozialdemokratischen Politik Schwedens. Vorausgesetzt, die Fonds plazieren ihr Kapital gezielt und strategisch, können sie in Zukunft den Machtpositionen privater Besitzergruppen entgegenwirken. Diese Möglich- 
keit erklärt auch den heftigen Widerstand von Seiten der SAF und anderer Wirtschaftsorganisationen.

\section{Das »schwedische Modell im Umbruch}

Wir haben gesehen, daß die Strategie der »Instrumentalisierung des Staates « während der $70 \mathrm{er}$ Jahre zu keinen grundlegenden Veränderungen im traditionellen Modell schwedischer So zialbeziehungen geführt hat, das auf Kooperation und Selbstregulierung beruht. Die gegenwärtigen Entwicklungen sind demgegenüber durch zwei Trends bestimmt: Zum einen sind auf Arbeitgeberseite verstärkt Bestrebungen sichtbar, das Tarifvertragssystem zu »japanisieren« und die bisherige Tradition der Kooperation mit den Gewerkschaften zu unterminieren. Das»schwedische Modell«der Kooperation in den Arbeitgeber- und Arbeitnehmerbeziehungen befindet sich also im Umbruch. Zum anderen sind angesichts der gesamten gegenwärtigen Wirtschaftsprobleme verstärkt Gegensätze zwischen der LO und der sozialdemokratischen Regierung aufgetreten; die Fortentwicklung des »schwedischen Modells« ist geschwächt. $\mathrm{Zu}$ diesen beiden Trends nun die beiden folgenden Ausführungen:

\subsection{Bestrebungen der Arbeitgeber, das Tarifvertragssystem zu »japanisieren"}

Während der letzten drei bis vier Jahre ist der ArbeitgeberverbandSAF in die Offensive gegangen, nicht nur, um die Arbeitnehmerfonds zu bekämpfen, sondern auch um das Tarifvertragssystem selbst zu verändern und es mehr an die Anforderungen einzelner Industriezweige und Unternehmen anzupassen. Darüber hinaus kann man von einer allgemeinen ideologischen Offensive sprechen, die darauf zielt, die Stellung des Individuums gegenüber dem Kollektiv herauszustreichen. Typisch hierfür ist die Kampagne »Setz aufDich selbst! «, deren wichtigste Zielgruppe arbeitslose Jugendliche sind. Die gleiche Ideologie liegt jenen individuellen Gewinnbeteiligungsmodellen zugrunde, die eine Reihe von Unternehmen, u.a. Volvo, als Gegenvorschlag in die Arbeitnehmerfonds-Debatte eingeführt haben.

In bezug auf das Tarifvertragssystem bestehen die Bestrebungen von SAF darin, die Bedeutung gewerkschaftlicher Interessenvertretung zu verringern und die Gewerkschaftseinheit zu unterminieren. Dies geht zum einen aus dem Verhalten von SAF während der Tarifrunde hervor, in denen man nicht ohne Erfolg einen Keil zwischen die Arbeiter- und Angestelltengewerkschaften treiben konnte, indem man dem PTK, dem Verhandlungsverbund der Angestellten der Privatindustrie, im Vergleich zur LO vorteilhaftere Tarifabkommen angeboten hat. Zum andern hat SAF seit der letzten Tarifrunde die Zielsetzung verfolgt, die zentralen Verhandlungen mit LO abzuschaffen. Die gegenwärtig laufende Tarifrunde über die Löhne des Jahres 1984 ist die erste seit 1955 (als die 1952 eingeführten zentralen Verhandlungen zeitweilig durch freie Verhandlungen auf der Ebene der Einzelgewerkschaften ersetzt wurden), die ohne zentrale Verhandlungen stattfindet. Ursprünglich hatte SAF selbst zentrale Verhandlungen durchgesetzt, mit der Absicht, dadurch die Zahl der Konflikte zu verringern, einheitliche Vertragsperioden zu erreichen und durch gleichartige Verträge die Arbeitgeberkonkurrenz um die Arbeitskräfte auf den Arbeitsmärkten zu dämpfen. ${ }^{36}$

SAF als hochdisziplinierte und zentralisierte Organisation änderte im Frühjahr 1982 seine Satzung, um den Mitgliedsorganisationen größeren Spielraum zu geben. Bis zu diesem Zeit- 
punkt hatte die Zentrale sich das Recht vorbehalten, Verträge, die die angeschlossenen Verbände eingehen wollten, gutzuheißen oder zu verwerfen. Innerhalb von SAF tonangebend für die neue, stärker dezentralisierte Strategie auf Arbeitgeberseite war der Metallarbeitgeberverband. Bereits während der Tarifrunde 1983 scherte man aus den zentralen Verhandlungen aus und schloß ein separates Abkommen mit der Gegenseite, der Metallarbeitergewerkschaft. Dies bedeutete den Anfang vom Ende der zentralen Verhandlungen. LO versucht zwar, in der gegenwärtigen Tarifrunde eine Koordinierung der Einzelgewerkschaften, doch steht dahin, inwieweit dies gelingt.

Ein Ereignis, das zweifellos zu einer Überprüfung des bisherigen zentralisierten Verhandlungsmodells durch die SAF beigetragen hat, war der große Arbeitskonflikt von $1980 .{ }^{37}$ Dieser bestand aus Punktstreiks (von Seiten der LO) und einer Großaussperrung (von Seiten der SAF). Im Organisationsbereich SAF-LO streikten rund 100000 Arbeiter, darüberhinaus waren 700000 zehn Tage lang ausgesperrt. Bereits 1979/80 hatte das Präsidium von SAF Verhandlungen auf Einzelverbandsebene erwogen, die Möglichkeit jedoch, ein schnelles Abkommen mit geringen Lohnerhöhungen zu erreichen, trug dazu bei, daß man am zentralen Modell festhielt. ${ }^{38}$ Das Ergebnis war jedoch der besagte Großkonflikt und aus ihm resultierende recht ansehnliche Lohnerhöhungen (ca. 7 \% gegenüber SAF's Ausgangsangebot von $0 \%)$. SAF sah sich dazu gezwungen, auf direktes Drängen des bürgerlichen Regierungschefs hin, den Tarifvorschlag der Schlichtungskommission zu akzeptieren. Der Unmut der Arbeitgeber richtete sich in erster Linie darauf, daß man meinte, die Organisationen der Angestellten des öffentlichen Dienstes hätten mit ihrer erfolgreichen Initiative in der Tarifrunde sowohl den Konflikt als auch den für die SAF unvorteilhaften Tarifvertrag verursacht.

Besonders der Arbeitgeberverband schwedischer Metallindustrieller hat in den letzten Jahren immer wieder hervorgehoben, daß die Exportindustrie und nicht der öffentliche Dienst bei den Löhnen richtungsweisend sein solle. ${ }^{39}$ Es wird zunehmend als ein Problem angesehen, $\mathrm{da}$ L Lohnangleichungen in anderen Sektoren automatisch erfolgen, wenn die Industriearbeiter durch höhere Akkordleistungen ihre Löhne zu erhöhen vermögen. Dies geschieht durch eine Reihe von »Nachfolgeklauseln«, welche die Löhne für praktisch alle Arbeitnehmergruppen an die Lohnbewegungen der Industriearbeiter anpassen. Stattdessen, so die Metallarbeitgeber, solle der Produktivitätsgewinn in erster Linie jenen zukommen, die ihn verursachen. So wurden sowohl die zentralen Verhandlungen, die in der Praxis eine Koordinierung des gesamten Arbeitsmarktes (das heißt nicht nur des Organisationsbereichs LO-SAF) mit sich geführt hatten, als auch die solidarische Lohnpolitik im LO-Bereich jetzt von den Metallindustriellen als Hindernis einer produktivitätsorientierten Lohnpolitik bezeichnet.

Stattdessen will man nun ein Verhandlungssystem, das vom Zahlungsvermögen der einzelnen Branchen und Unternehmen, dem Qualifikationsgrad der Arbeit und der persönlichen Leistung ausgeht. Auch für Arbeiter soll im Rahmen des Tarifvertrages die Möglichkeit von individuellen Lohnzuwächsen geschaffen werden. Die Metallarbeitergewerkschaft hat von sich aus ebenfalls die lohnleitende Rolle der Industrie - im Gegensatz zum öffentlichen Dienst - betont und in Frage gestellt, daß andere Gruppen für den Lohnanstieg der Industriearbeiter eine automatische Angleichung erhalten. ${ }^{40}$ Der Einzelvertrag zwischen dem Arbeitgeberverband schwedischer Metallindustrieller und der Metallarbeitergewerkschaft von 1983 veranlaßte ersteren zu der Schlußfolgerung, »daß ein gemeinsames Interesse auf Branchenniveau darin vorhanden ist, eine solche 'Japanisierung' der Beziehungen zwischen den Partnern zu erreichen. ${ }^{41}$ Die Metallindustriellen weisen in diesem Zusammenhang auch darauf hin, daß die neuen Techniken mit der immer weiter fortschreitenden Verwendung u.a. 
von Datenverarbeitungsgeräten und Robotern die Voraussetzungen für neue Formen der Arbeitsorganisation schaffen, und daß deshalb Vertragsformen erforderlich seien, die dieser Entwicklung adäquat seien und »eine positive Zusammenarbeit am Arbeitsplatz« ermöglichen. Unter diesem Aspekt darf man das Interesse von SAF und dem Verband schwedischer Metallindustrieller an den 1982 bis '83 eingegangenen Umsetzungsvereinbarungen sehen.

Der Wunsch nach neuen, produktivitätsfördernden Lohnsystemen hängt auch mit der abnehmenden Bedeutung der Akkordarbeit zusammen. 1964 hatten nur 2,5\% der Arbeiter in der Maschinenbau- und metallverarbeitenden Industrie einen festen Monats- oder Wochenlohn gegenüber 9 bis $10 \%$ aller Industriearbeiter $1973 .{ }^{42}$ Seither hat ihr Anteil ständig zugenommen.

Ein anderes Motiv dafür, das System der Zentralverhandlungnen aufzugeben, besteht darin, damit das Risiko staatlicher Einkommenspolitik zu mindern. ${ }^{43}$ Den Hintergrund dafür bildet die Tatsache, daß die Steuerpolitik und andere staatliche Maßnahmen mit der Zeit immer mehr in die Tarifverhandlungen einbezogen wurden. Überhaupt sind koordinierte Verhandlungen immer komplizierter geworden, wobei das Paar LO und SAF an Bedeutung verlor zugunsten der Regierung, dem Verhandlungsverbund der Angestellten der Privatindustrie sowie der Gewerkschaften der Angestellten des öffentlichen Dienstes. In dieser Situation hat SAF sich entschlossen, den Schwerpunkt des Verhandlungssystems nach unten zu verlagern. Durch die Schwächung der zentralen Ebene besteht die offensichtliche Gefahr einer zunehmenden Spaltung unter den Arbeitnehmern und innerhalb des zentralen Gewerkschaftsbundes. Unter anderem wird es schwierig, eine solidarische Lohnpolitik zu verwirklichen.

\subsection{Zunebmen der Gegensätze im Verbältnis von Gewerkschaftsbewegung und Sozialdemokratie}

Die gestiegenen Gegensätze zwischen LO und PTK und die Spannungen innerhalb von LO, die zuletzt in dem Separatabkommen der Metallarbeitergewerkschaft mit dem Metallarbeitgeberverband zum Ausdruck gekommen waren, müssen vor dem Hintergrund des während der 80er Jahre geschrumpften »Lohnspielraums« und der Rolle, welche die Veränderung der Steuerskala für die Reallohnentwicklung(Schweden ist ein Land mit hohen Marginalsteuern) gespielt hat, gesehen werden. Die wachsenden Wirtschaftsprobleme in bezug auf die staatliche Verschuldung und die Arbeitslosigkeit haben auch zu verstärkten Gegensätzen zwischen LO und der sozialdemokratischen Regierung geführt. Natürlich vertritt LO eine stärker nachfragestimulierende Wirtschaftspolitik und setzt sich in höherem Grad für die Beschäftigungslage der im öffentlichen Dienst Beschäftigten ein, als die für die Staatsfinanzen verantwortliche Regierungspartei.

Die sozialdemokratische Partei betont die Anfälligkeit Schwedens für Veränderungen des internationalen Wirtschaftssystems und befürwortet eine offensivere Strukturpolitik - unter Mitwirkung der Privatwirtschaft - gegenüber der bisherigen Strukturpolitik der bürgerlichen Regierung, die den von der Krise erfaßten Industriezweigen massive Staatssubventionen hat zukommen lassen. ${ }^{44}$ Andere »Rezepte« sind die Kürzung der öffentlichen Ausgaben und im Vergleich zu früher geringere Lohnsteigerungen. LO setzt demgegenüber die ganze Hoffnung auf eine nachfragestimulierende Politik.

Sowohl LO als auch die Sozialdemokratische Partei haben ein eigenes ökonomisches Handbuch herausgegeben, und aus ihnen wird die unterschiedliche ökonomisch-politische Aus* 
richtung sehr deutlich. Das LO-Buch Påkurs mot Framtiden des LO-Ökonomen Claes-Erik Odhner et al. gilt als Gegenveröffentlichung zum »Parteibuch«I Bräcklig Farkost des Sozialdemokraten Ahlqvist. ${ }^{45}$ Ahlqvists Buch ist eine Auftragsarbeit für die Parteileitung. Versehen mit einer umfassenden Leseanleitung wird dieses Handbuch gelesen und diskutiert in den Mitgliederorganisationen der Partei. Stig Malm, Sprecher der LO, hat das Diskussionsklima dort wie folgt charakterisiert: "Ahlqvist und Milton Friedman können einander die Hand reichen. Ihre ökonomischen Vorstellungen passen möglicherweise für Chile, aber nicht für Schweden." (Dagens Nyheter vom 31.10.1983)

Einer der Vorschläge Ahlqvists sieht nicht mehr und nicht weniger vor als die Aufgabe des Systems freier Tarifverhandlungen zugunsten eines Systems »effektiver Einkommenspolitik«. ${ }^{46}$ Demzufolge soll von Seiten des Staates die Höhe des Lohnzuwachses festgelegt werden. Arbeitgebern, welche diese Höchstgrenze überschreiten, wird eine Strafsteuer auferlegt. Falls dieser Vorschlag verwirklicht werden sollte, könnten sich die Gewerkschaften ebensogut gleich auflösen, da sie dann ihrer wesentlichen Funktionen beraubt wären.

Die unterschiedlichen wirtschaftspolitischen Auffassungen von LO und Partei drücken natürlich ihre verschiedenen Stellung im Verhältnis zu Staat und Arbeiterklasse aus. Doch auch innerhalb der Partei sind die Meinungen geteilt. Viele Arbeiter sind Parteimitglieder auf Grund der kollektiven Mitgliedschaft, die von den meisten an der LO beteiligten Gewerkschaften praktiziert wird. Wie Stichprobenuntersuchungen (vgl. LO-Zeitung Nr. 7, 1979 und Nr. 4, 1982) zeigen, liegt die beschlußfassende Macht in der Partei heute jedoch bei Angestellten, Beamten und Berufspolitikern.

In dieser Situation scheint es kaum möglich, daß über die Achse Gewerkschaftsbewegung Sozialdemokratie neue Ideen zur Bekämpfung der Wirtschaftskrise entwickelt werden, die Arbeitnehmerfonds einmal ausgenommen. Im Unterschied etwa zur Bundesrepublik glaubt man auf Seiten der Gewerkschaften zum Beispiel nicht, daß Arbeitszeitverkürzung oder Arbeitsteilung(Job-sharing) ein Mittel sein könnte, die Arbeitslosigkeit zu mindern. Im Prinzip ist man positiv eingestellt gegenüber einer Verkürzung der Arbeitszeit, betrachtet es jedoch nicht als aktuell, in nächster Zeit die Arbeitswoche allgemein zu verkürzen ${ }^{47}$ Man fürchtet, daß kürzere Arbeitszeit noch weiter gesenkte Reallöhne mit sich führen könnten. Es ist typisch, daß die schwedische Metallarbeitergewerkschaft sich der Stimme enthielt, als der internationale Metallarbeiterbund über die Arbeitszeitverkürzung abstimmte. ${ }^{48}$

Was Mitbestimmungsfragen und Fragen der Arbeitsumwelt angeht, hat man seit Ende der sechziger Jahre sicherlich Fortschritte gemacht. Dennoch haben Umstrukturierungen und Entlassungen überdeutlich gemacht, daß der Besitz der Produktionsmitttel nach wie vor das entscheidende Machtinstrument darstellt. Weitere Fortschritte für die Arbeitnehmer setzen wesentliche Veränderungen in dieser Hinsicht voraus. Möglicherweise können die Arbeitnehmerfonds den Beginn einer entsprechenden Entwicklung darstellen. Dazu ist aber im Vergleich zu heute ein wesentlich offensiveres Auftreten der Arbeiterbewegung vonnöten. Im Augenblick befinden sich stattdessen die Arbeitgeber auf dem Vormarsch. Ob»Japanisierung« oder »Radikalisierung« die kommenden Jahre bestimmt, bleibt offen.

Übersetzung aus dem Schwedischen: Ulrich Jürgens, Claudius H. Riegler und Trangott Koch 
Tabelle 1. Gewerkschaftlicher Organisationsgrad für Arbeiter und Lohnabhängige in zwölf Ländern.

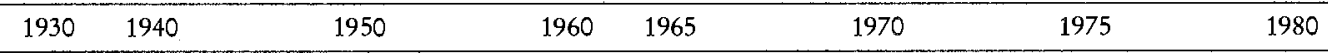

SCHWEDEN ${ }^{1}$

Arbeiter (1)

$45 \quad 66$

Arb. (obne Agrarsektor)

Arbeiter (2)

$\begin{array}{llll}55 & 73 & 76 & 78 ; 76 \\ & 79 & 80 ; 78\end{array}$

Lohnabhängige (1)

$41 \quad 58$

67

$80 ; 78$

Lohnabhängige (2)

$68 ; 68$ ca. 71

$\begin{array}{lll}44 & 56 \\ 67 & 73 \\ & 54 \\ 37 & 46 & \\ & 51\end{array}$

65

78

61

61
53

(2)

58

,

$\begin{array}{ll}69 & 6 \\ 77 & 74 \\ 67 & 6 \\ 62 & 62 \\ 62 & 63\end{array}$

$\begin{array}{ll}69 & 7 \\ 74 & 72 \\ 69 & 74 \\ 62 & 62 \\ 63 & 6\end{array}$

$\begin{array}{ll}71 & 80 \\ 72 & 81 \\ 74 & 76 \\ 62 & 67 \\ 64 & 69\end{array}$

\section{NORWEGEN ${ }^{3}$}

Arbeiter

$\begin{array}{ll}31 & 63^{4} ; 54 \\ 33 & 69^{4} \cdot 59\end{array}$

70

$74^{5} \cdot 71$

73

85

85

86

Arb. (obne Agrarsektor)

Lohnabhängige (1)

Lohnabhängige (2)

$\begin{array}{cc}33 & 69^{4} ; 59 \\ (23) & 47^{4} ; 40\end{array}$

75

(52)

$\begin{array}{rr}74^{5} ; 71 & 73 \\ 78^{5} ; 73 & 74 \\ 64 & 66\end{array}$

74

74

64

61
55

65

ÖSTERREICH ${ }^{6}$

Industriearbeiter

Lohnabhängige

38

74

62

77

63

73

62

59

74

\section{BELGIEN $^{7}$}

Arbeiter (1)

a Lohnabhängige (1)

33

28;35

56
52

74

57

68
55

$61 \quad 60$

74

61

65

66

66
69

71

AUSTRALIEN $^{8}$

Lohnabhängige (1)

29

60

55

54
56

51

51
50

54

$\begin{array}{lll}44 & 40 & 56 \\ 53 & 48 & 58\end{array}$

56
58

58

I

(50

। 56


GROSSBRITANNIEN

$\begin{array}{lllll}\text { Arbeiter } & 26 & 34 & 48 & 50\end{array}$

Lohnabhängige

$26 \quad 33$

$50 \quad 49$

$52 ; 56^{9}$

$47 \quad 58$

DEUTSCHLAND/BRD ${ }^{10}$

Arbeiter

Arb. (ohne Agrarsektor)

Lohnabhängige

$44 \quad 35 ; 38^{11}$

$47 \quad 38 ; 41^{11}$

$38 ; 41^{11}$
$33.37^{11}$

35

43

48

35

$33^{11} ; 35$

$33^{11} ; 35$

$31^{11 ; 32}$

39

39

33

NIEDERLANDE ${ }^{12}$

Lohnabhängige (1)

30

31

40

39

37

37

39

38

Lohnabhängige (2)

ITALIEN $^{13}$

Lohnabhängige (1)

Lohnabhängige (2)

Lohnabhängige (3)

$44^{14} ; 34$

$36^{15}$
$32^{15}$

$46^{16}$

$41^{16}$

31

$\begin{array}{r}53 \\ +\quad 47 \\ \hline\end{array}$

FRANKREICH ${ }^{18}$

Lohnabhängige (1)

$9 \quad 41^{19} ; 21^{20}$

$46^{21} ; 32^{22}$

$22^{23}$

$18^{24}$

23

25

25

Lohnabhängige (2)

USA

Arbeiter ohne agrar.

und Dienstleitungsber.

Lohnabhängige (1) ${ }^{26}$

Lohnabhängige (2) ${ }^{26}$

$\begin{array}{cc}15 & 31 \\ 11 & 22 \\ 12 & (27)\end{array}$

$\begin{array}{cccc}\text { ca. } 55 & / & 55^{25} & 51^{25} \\ 31 ; 33^{27} & & 29 & 28 \\ 34^{27} & & 31 & 28\end{array}$

$51^{25}$
28
28

28

$51^{25}$
29
30

$47^{25}$

28

29

$40^{25}$

Anm.: Im Allgemein. Stichtag 31.12. / markiert eine Diskontinuität. Quelle: Anders Kjellberg Facklig organisering i tolv länder/Gewerkschaftliche Orga-

nisierung in zwölf Ländern), Arkiv Verlag, Lund 1983, $536 \mathrm{ff}$ 
1 Arbeiter (1) und Lohnabhängige(1) nach der Volkszählung. Arbeitslose eingerechnet, pensionierte Gewerkschaftsmitglieder dagegen unberücksichtigt. Arbeiter (2) und Lohnabhängige (2) sind den Lebensstandarduntersuchungen entnommen und gelten für 1968, 1974 und 1981.

2 Arbeiter (1) und Lohnabhängige (1) bzw. Arbeiter (2) und Lohnabhängige (2) bezieht sich auf verschiedene Berechnungsweisen. Arbeitslose eingerechnet.

3 Nur LO-angeschlossene Lohnabhängige berücksichtigt für die Jahre 1930-50. Lohnabhängige (2) läßt pensionierte LO-Mitglieder aus. Arbeitslose mitgerechnet.

4 Gilt 1939.

5 Gilt 1954.

6 Bei den Industriearbeitern (Bau- und Grubenarbeiter sowie Arbeitslose einbezogen) gilt die Zahl unter 1950 für 1952 und die unter 1960 für 1962.

7 Arbeiter(1) und Lohnabhängige (1) bezieht sich auf den FGTB und den CSC, während Lohnabhängige (2) auch den CGSLB umfaßt. Arbeiter(1) berücksichtigt nach 1950 nicht mehr die Arbeiter im Offentl. Dienst.

8 Die obere Zahlenreihe stammt von George Sayers Bain \& Robert Price (siehe Profiles of Union Growth, Basil Blackwell, Oxford 1980), die untere aus der offiziellen Statistik.

9 Gilt für United Kingdom, also einschl. Nordirland. Die Ziffer für Arbeiter 1975 gilt für 1974.

10 Alle drei Zahlenreihen schließen Arbeitslose ein und lassen nach 1950 DGB-Pensionäre unberücksichtigt. Nicht-agrare Arbeiter sind ohne Land- und Forstarbeiter gerechnet (1930 auch ohne Fischerei). In der Zahlenreihe für Lohnabhängige tritt $z$ wischen 1970 und 1975 eine größere Veränderung ein, als Soldaten ab 1971 zu den Lohnabhängigen gerechnet werden.

11 Die zweite Zahl unter 1950 gilt für 1951 und die erste unter 1970 für 1969.

12 Lohnabhängige (2) ist nach einer neuen Methode berechnet. Alle Zahlen umfassen Arbeitslose und schließen nach 1950 Pensionäre aus. Lohnabhängige (1) unter 1940 gilt für 1939.

13 Lohnabhängige (1) schließt im Unterschied zu den beiden anderen Zahlenreihen Pensionäre cin. Lohnabhängige (3) umfaßt nur die Organisationen CGIL und CISL. Alle Ziffern schließen Arbeitslose ein.

14 Gilt 1947.

15 Gilt.1968.

16 Gilt 1972.

17 Gilt 1979.

18 Die erste Zahlenreihe gilt 1930-40 für die Organisationen CGT + CGTU + CFTC und 1950-75 für CGT + CFTC/CFDT + FO + FEN. Die zweite Reihe umfaßt darüber hinaus CGC und CFTC(M). Seit 1960 sind CGT-Pensionäre weggelassen. Ab 1970 nur regulär bezahlende CFDTMitglieder.

19 Gilt 1937.

20 Gilt 1939.

21 Gilt 1946.

22 Gilt 1951.

23 Gilt 1962.

24 Gilt 1968.

25 Die Ziffer unter 1965 gilt für 1964, unter 1975 für 1974 und unter 1980 für 1978.

26 Beide Zahlenreihen umfassen nicht-agrar. Lohnabhängige, die erste nach NBER, die zweite nach BLS. Die ungenügende Zuverlässigkeit der BLS-Ziffer für 1940 wird durch die Klammer markiert. Ab 1970 schließen die BLS-Zahlen freistehende Berufsorganisationen aus dem Öffentlichen Dienst ein.

27 Gilt 1953. 


\section{Abkürzungen:}

LO Landsorganisationen i Sverige (Schwedischer Gewerkschaftsbund)

PTK Privattänstemannakartellen (Verhandlungskartell der Privatangestellten)

SACO-SR Akademikerorganisationen SACO-SR (Zentralverband der Akademiker)

SAF Svenska Arbetsgivareföreningen (Zentralverband Schwedischer Arbeitgeber)

TCO Tjänstemännens Centralorganisation (Zentralverband der Angestellten und Beamten)

\section{Anmerkungen}

1 Arbeitslose im Verhältnis zu der arbeitsfähigen Bevölkerung nach den Arbeitskraft-Untersuchungen (AKU). Siehe: Statistik årsbok 1984, Stockholm 1983, s 184. In absoluten Ziffern waren 1978 94000 Personen und 1982137000 Personen arbeitslos. Rechnet man Beschäftigte in Arbeitsbeschaffungsmaßnahmen, Arbeitsmarktausbildung, geschützten Werkstätten (»Samhällsföretag«) und Angestellte mit Lohnsubvention dazu, dann wachsen die Zahlen auf 2330001978 und 278000 1982.

2 Eine Übersicht über die Organisation der Arbeitgeber in verschiedenen Ländern gibt: Kjellberg Facklig organisering $i$ tolv länder, Arkiv Verlag, Lund 1983, in Kap. 4.

3 Veckans affärer nr 271983 s 40

4 Vergleiche Edward Shorter \& Charles Tilly: Strikes in France Cambridge University Press, London 1974 , s $318-325$ und 333.

5 Organisation \% Demokrati. Rapport till LO 80 - Utredningens demokratigrupp, LO, Stockholm 1981 und Vem är aktiv i facket? En undersökning fråan LO 80 - utredningen, LO, Stockholm 1981.

6 Organisation \& Demokrati ibid s 54

7 Anders Kjellberg 1983, Abschnitt 5.4. und 7.1.

8 Ibid s 169

9 Bo Rothstein "AMS som socialdemokratisk reformbyråakrati « Arkiv für studier i arbetarrörelsens bistoria nr 181980

10 Kjellberg 1983 Abschnitt 7.5.

11 Kjellberg 1983 Abschnitt 7.5.

12 Anders Kjellberg »Fråan industriell demokrati till medbestämmande - fackliga utvecklingslinjer 1917-1980 « Arkiv für studier i arbetarrörelsens bistoria nr 21-22 1981

13 Spontaneität und Massenaktion im „Woblfabrtsstaat«. Die schwedischen Streiks im Winter 1969/70. Frankfurt 1970

14 Kjellberg 1981 s $75 \mathrm{f}$

15 Metallarbetaren $\mathrm{nr} 411983 \mathrm{~s} 15 \mathrm{ff}$

16 Olle Hammarström »Medbestämmande 1977-79 - en översikt« in: Tre åar med MBL, Publica, Stockholm 1980

17 Horst Hart Vad betyder medbestämmandet för arbetsgivaren?, Sociologiska institutionen Göteborg 1980 s $33 \mathrm{ff}$

18 LO-tidningen $\mathrm{nr} 14-161982 \mathrm{~s} 8 \mathrm{f}$

19 In Form eines Unternehmensvertrags, abgeschlossen am 25.11.1983. Siehe Dagens Nyheter 1.12.1983

20 Metallarbetaren nr $411983 \mathrm{~s} 15 \mathrm{ff}$

21 Für eine Übersicht der Studien von LO zum Themenkreis der Humanisierung des Arbeitslebens vergl.: Vad bänder med arbetsmiljön? Rapport om LO-medlemmamas och skyddsombudens erfarenheter (von Erik Bilinder u.a.), LO, Stockholm 1981 s $9 \mathrm{ff}, 19 \mathrm{ff}$ und $174 \mathrm{ff}$ 
22 Lars Lundberg Fråan lag till arbetsmiljö, Liber Verlag, Malmö 1982 s 46 ff, 74 ff und 80 ff

23 Ibid $s 65$ und $126 \mathrm{ff}$

24 Ibid s $122 \mathrm{ff}$

25 Ibid s $130 \mathrm{ff}, 146$ und 152

26 Siche z.B. Lag \& Avtal nr 923.11 .1983 s 6

27 Ibid s 12

28 Ibid s $11 \mathrm{f}$

29 Dies ist weder bei Lundberg noch bei Kelman, der die schwedischen und die US-amerikanischen Arbeitsschutzbehörden vergleichend untersucht hat, beachtet worden.

Vergleiche Lundberg ibid und Steven Kelman Regulating America, Regulating Sweden: A Comparative Study of Occupational Safety and Health Policy, The MIT Press, Cambridge Mass. 1981.

Kelman stellt der schwedischen, auf Zusammenarbeit ausgerichteten Behördentätigkeit die mehr legalistische und strafandrohende in den USA gegenüber. Während der behandelten Periode $1970-$ 1976 tritt die amerikanische Behörde, OSHA (gegrïndet 1970), unter dem republikanischen Präsidenten Nixon wesentlich bestimmter gegenüber den Arbeitgebern auf als ihre schwedische Entsprechung unter einer sozialdemokratischen Regierung. Auch die amerikanischen Grenzwerte u.a.m. waren vorteilhafter für die Arbeiter, verglichen mit Schweden.

30 Kjellberg 1983 Kap 3

31 Vad händer med arbetsmiljön? ibid s $140 \mathrm{ff}$

32 Ibid s $156 \mathrm{ff}$

33 Ibid $\mathrm{s} 152 \mathrm{f}$

34 Ibid s $153 \mathrm{f}$

34 a Die Parlamentsentscheidung vom 21. Dezember 1983 über die Arbeitnehmerfonds sieht vor, daß zu Beginn des Jahres 1984 von der Regierung die Aufsichtsräte für fünf Regionalfonds ernannt werden. In ihnen werden die Vertreter der Arbeitnehmer die Majorität haben. Die Fonds werden jährlich bis einschließlich 1990 für 2 Mia. schwedische Kronen Aktien erwerben. Die Finanzmittel dafür kommen erstens aus einer »Arbeitgeberabgabe - berechnet als Prozentsat $z$ von der Lohnsumme -, zum zweiten aus einer speziellen Gewinnsteuer. Auf Anfrage sind $50 \%$ der Stimmrechte über ein Unternehmen, die bei dem Fonds liegen, an die örtliche Gewerkschaftsorganisation dieses Unternehmens zu übertragen. Der Kauf von Aktien durch einen Arbeitnehmerfonds darf $8 \%$ der Stimmrechte der Aktionäre des Unternehmens nicht überschreiten, der von den Arbeitnehmerfonds insgesamt erworbene Anteil darf $50 \%$ nicht überschreiten.

35 Zur Entwicklung der Diskussion über Arbeitnehmerfonds bis 1978 siehe Erik AAsard LO och löntagarfondsfråagan, Rabén \& Sjögren, Stockholm 1978. Siehe auch: Sandra L. Albrecht \& Steven Deutsch »The Challenge of Economic Democracy: The Case of Sweden« Economic and industrial democracy vol $4 \mathrm{nr} 31983$

36 Siehe das Interview mit dem Verhandlungschef von SAF, Lars-Gunnar Albåage, Dagens Nyheter 8.11 .1983

37 Eine Dokumentation zum Großkonflikt 1980 findet sich in: Anders Broström (Red.) Storkonflikten 1980, Arbetslivscentrum, Stockholm 1981. Vergleiche auch dic aus Arbeitgebersicht geschricbene Schrift von Olle Bolang Utslagen. Den svenska modellen efter 1980 åars storkonflikt, SNS, Stockholm 1980.

38 Interview mit dem Vorsitzenden von SAF, Olof Ljunggren, Dagens Nybeter 22.9.83

39 Siehe die Leitartikel in: Verkstäderna nr 3, 4 und 91983.

40 Vergleiche z.B. das Interview mit dem Vorsitzenden des Metallarbeiterverbandes Leif Blomberg in: LO-tidningen $\mathrm{nr} 19-201983 \mathrm{~s} 11$.

41 Verkstäderna nr $41983 \mathrm{~s} 7$

42 Thommy Svensson Fråan ackord till måanadslön, Svenska Varv AB, Göteborg 1983, s 333 f

43 Siehe den Vortrag des SAF-Vorsitzenden Olof Ljunggren 27.11.1982 in: Inför avtal 83, SAF, Stockholm 1983.

44 Eine Studie von Bo Carlsson am Forschungsinstitut der Industrie zeigt schwedische Industrie- 
Subventionen sowohl in Umfang als auch in ihrer Selektivität in internationaler Spitzenposition. In Prozent des Veredlungswertes der Industrie betrugen die Subventionen in Schweden 16.0\% (1979), in Finnland 10,9\% (1979), in Norwegen 7,6\% (1979), in Italien 7,1 \% (1978), in der Bundesrepublik Deutschland 4,0\% (1980) und in Großbritannien 3,6\%(1979-80). Siehe Bo Carlsson "Det svenska industristödet $\mathrm{i}$ internationell jämförelse«

Ekonomisk debatt nr 71983.

45 Beide Bücher sind erschienen im Tidens Verlag, Stockholm 1983

46 Ahlquist ibid., S. $160 \mathrm{f}$

47 Vergleiche den Bericht Lönepolitik för 80-talet, LO, Stockholm 1981, S. $109 \mathrm{ff}$

48 Bertil Edin vom internationalen Sekretariat der Gewerkschaft motiviert diesen Standpunkt auf folgende Weise: »Es gibt gegenwärtig andere Fragen, die für die Arbeitnehmer wichtiger sind. Sieben Jahre lang haben wir eine Senkung des Lebensstandards hinnehmen müssen. Weitere Reallohnverluste können von den Arbeitnehmern schwer ertragen werden. (...) Die Statistik aus Ländern, die kürzere Arbeitszeit eingeführt haben, nämlich Frankreich, Belgien und England, zeigt keine Änderung der Arbeitslosenziffern. (...) Aber wir unterstützen den Kampf der Westdeutschen (Metallarbeiter) für kürzere Arbeitszeit. Können sie Probleme für die Deutschen lösen, dann stehen wir natürlich hinter deren Forderungen. « (Metallarbetaren Nr. 48/1983, Seite 5) 